\title{
Integration of a suite of sensors in a wireless health sensor platform
}

\author{
Pepijn van de Ven*, Alan Bourke*, Carlos Tavares ${ }^{\dagger}$, Robert Feld $^{\ddagger}$, \\ John Nelson*, Artur Rocha ${ }^{\dagger}$, and Gearóid Ó Laighin ${ }^{\S}$ \\ ${ }^{*}$ Dep. of Electronic \& Computer Engineering \\ University of Limerick, Ireland \\ Email: pepijn.vandeven@ul.ie \\ $\dagger$ INESC Porto, Portugal \\ ‡Corscience GmbH \& Co. KG Erlangen, Germany \\ $\S$ Dep. of Electrical \& Electronic Engineering \\ National University of Ireland, Galway, Galway, Ireland
}

\begin{abstract}
In this paper we discuss the development and clinical evaluation of a wireless platform for health signs sensing. The sensors measure physical activity, ECG, blood oxygen saturation, temperature and respiratory rate. An important aspect of the approach is that the sensors are integrated into one waist-worn device. A mobile phone collects data from this device and uses data fusion in the scope of a decision support system to trigger additional measurements, classify health conditions or schedule future observations. In these decisions, the user's current physical activity plays an important role as the validity of many health signs measurements is strongly related to physical activity. Due to the integration of the sensors and the use of data fusion it is possible to accurately identify health risks and to react promptly. During clinical trials, for which proper ethical approval was obtained, the system was used by healthy elderly volunteers in Limerick (Ireland) and Ancona (Italy). Results of these trials are also discussed in this paper.
\end{abstract}

\section{INTRODUCTION}

Throughout the world, population characteristics are changing at an alarming rate [1]. For example, world-wide the number of citizens 60 years or over was estimated to be 688 million in 2006. By 2050 their numbers will have increased to almost two billion. This demographic trend is largely due to the vast improvements in health care and ironically, it is exactly this important aspect of our society that will suffer most. Recently the European FP6 project CAALYX [2] (Complete Ambient Assisted Living Experiment) was successfully completed. In this project with partners from 6 different European nations, a suite of sensors in a body area network is used to gauge the medical condition of elderly users, thus lessening the strain on traditional health services. The physiological parameters measured in the CAALYX system are: blood pressure (BP), heart rate $(\mathrm{HR})$, blood oxygen saturation $(\mathrm{SpO} 2)$, temperature, respiratory rate and weight. A common problem with the ageing is an increase in the incidence of falls due to a general deterioration in motor control and in the integrity of musculoskeletal structures. The results of a fall can be dramatic, leading to long hospitalisation and, frequently, death as a direct or indirect consequence of the fall [3]. For this reason, and as recent physical activity is an important factor in determining whether or not it is suitable to perform a certain measurement at a particular time, a falls and mobility monitor was integrated with an ECG, $\mathrm{SpO} 2$ and temperature sensor into one device, dubbed the "wearable light device" (WLD). The weight scales and blood pressure meter are stand-alone devices that communicate with the system through a pc application. The elderly people are equipped with the WLD, which wirelessly relays the gathered data to a $3 \mathrm{G}$ enabled phone. The data are then relayed to a server, which can be consulted by health practitioners. The server relays events and alerts automatically to a care centre specialised in processing these events and alerts. Figure 1 illustrates the CAALYX system. This paper

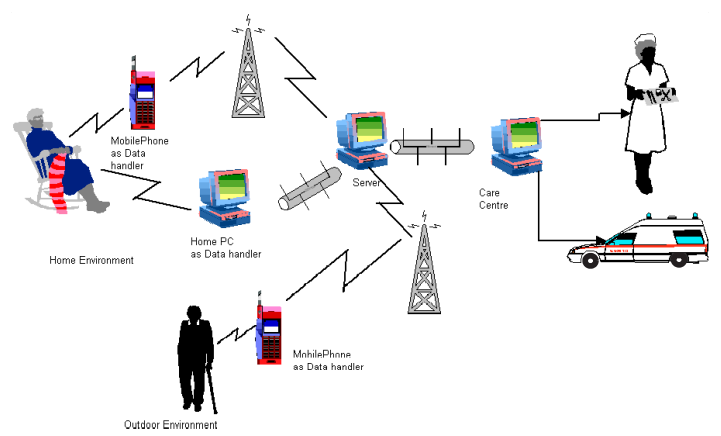

Fig. 1. The CAALYX system

discusses the design and technical and clinical tests performed with the WLD. Due to space constraints the other parts of the CAALYX system will not be further discussed in this paper. In section II the design and development of the WLD, the sensors it constitutes and the software necessary to control the device are described. Section III details the clinical trials performed with the sensors and conclusions can be found in section IV.

\section{DESIGN OF THE WIRELESS HEALTH SENSOR PLATFORM}

\section{A. Hardware}

The wireless health sensor platform consists of an ECG monitor, $\mathrm{SpO} 2$ sensor, temperature sensor and fall and mobility sensor. By combining measurements from the ECG monitor 


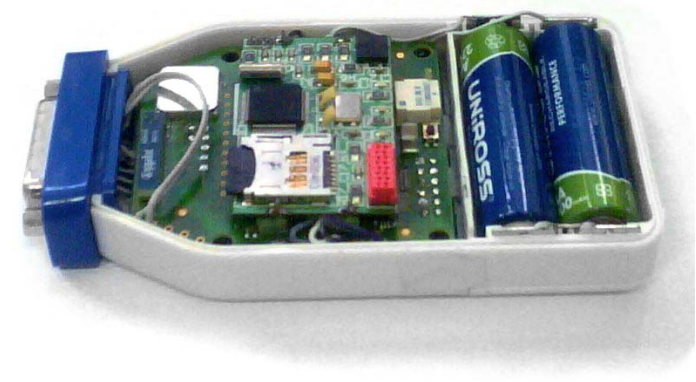

Fig. 2. WLD hardware

and the $\mathrm{SpO} 2$ sensor, a measure of the respiratory rate is obtained indirectly. The thus obtained physiological parameters can be used to diagnose a wide range of health issues. To ensure a high quality of gathered data, mobility information is used to schedule the measurements. The WLD hardware is depicted in figure 2 .

1) ECG sensor: The ECG sensor consists of an amplification stage with integrated electrode contact measurement functionality. The ECG waveform is recorded with a resolution of 24 Bits and a sample rate of $1 \mathrm{kHz}$ in order to obtain high quality signals as a basis for further processing. To suppress artifacts and reduce base line drift the one channel ECG uses an additional third electrode. The raw ECG signal is filtered to reduce the always present $50 / 60 \mathrm{~Hz}$ noise caused by the mains frequency. Furthermore a bandpass filter is applied to reduce several other noise sources. After filtering, the processed signal is fed into the r-wave detector. The resulting time stamps of the r-waves are used to calculate the heart rate with high precision. In order to suppress false readings due to artifacts, the WLD performs a plausibility analysis of the measured heart rate data and if a measurement is not considered valid, a zero measurement is sent to indicate that a measurement indeed has taken place. Those invalid measurements are filtered by the software on the N95 and only the remaining valid measurements are transmitted to the server. A further analysis of the ECG signal is used to extract features required to determine the respiratory rate (RR). Those are:

- The energy of the r-wave

- Variations in heart rate

- Variations in Pulse Transit Time (PTT)

The respiratory rate is calculated from different signals and the results are weighted for their quality and then a selection is combined to the output. As the signals apart from the ECG (PPG, PTT) depend on the finger to be in the finger clip, in most cases only the ECG was used for calculation. The RR calculation is very sensitive to artifacts in the ECG as the algorithm it is based on has to extract very subtle features from the ECG signal. Therefore a strict classification for validity is done with the resulting values and only those gained from stable signals of high quality are labelled as valid. Under laboratory conditions it was observed that only when a person is sitting and not speaking or moving energetically, a reliable output can be gathered.

2) Blood Oxygen Saturation: A pulse oximeter is integrated in the WLD and enables the non invasive determination of the blood oxygen saturation. For the measurement process the absorption of light of two different wavelengths through the finger is determined. The patient wears a flexible finger clip that has the light sources and detectors integrated. In addition to the $\mathrm{SpO} 2$, a photoplethysmograph (PPG) is recorded and used as a source of information for the respiratory rate detection. The processing of the recorded signal is complex and therefore is performed by a separate micro controller. Through a combination of the ECG and the PPG data, the pulse transit time can be determined. This is the time a pulse wave takes to travel from the heart to an extremity (i.e. the finger with the clip here). The WLD classifies the state of the sensor and the reliability of the measurements in several ways and is capable of recognizing and reporting a number of problems (e.g. too much ambient light and motion artifacts). Importantly only when a finger is detected to be inserted and the sensor has done a first analysis of the pulse waves and a self calibration is performed, measurements classified as valid are delivered.

3) Temperature: The temperature is measured with a skin surface sensor that uses a temperature dependent resistor that is integrated in a flat disc. The sensor was positioned in skin fold in the groin area of the user. The resulting measurement is corrected with calibration parameters in the main processor of the WLD. A new temperature value is obtained every second.

4) Fall and Mobility: The fall sensor is based on the measurement of acceleration in three degrees of freedom. The algorithms run on a Texas Instruments MSP430F1611. This processor was chosen for its low power consumption, sizeable memory (48kB flash with 256B user flash and 10kB RAM) and relatively high speed $(8 \mathrm{MHz})$. The MSP430F1611 is equipped with a 16 bit RISC processor, 3 DMA channels, a 12 bit ADC converter with 16 channels, a 12 bit DA converter, a hardware multiplier, 2 independent timers, 2 USARTs which can implement UART, SPI or I2C communication and a watch dog. The Freescale MMA7261QT tri-axial accelerometer is a low cost accelerometer with selectable outputs between $\pm 2.5-10 g^{1}$. The principle of operation is based on sensing changes in capacity due to flexing of moveable beams under the influence of accelerations. With a power consumption of $500 \mu \mathrm{A}$, the sensor is ideally suited for low-power applications. The measured accelerations are filtered using on-board single pole switched capacitor filters and can be directly fed to an AD converter.

5) Geo referencing: Every minute, the GPS receiver in the mobile phone will attempt to obtain a GPS fix. This period was defined after making a trade-off between power efficiency

\footnotetext{
${ }^{1} g$ Is the gravitational constant in $\frac{m}{s^{2}}$.
} 
and the required precision with which the location of the elder needs to be known. As elderly users will often be in their own homes where it will be difficult to obtain a reliable GPS fix, a mechanism to detect this situation was included in the CAALYX system. Upon detection of a wireless link with hardware in the home, the N95 notified the system of this fact thus offering an alternative positioning method.

\section{B. Software}

The mobile phone software was built on J2ME (Java for mobile) technology, which is available for the majority of commercial mobile phones including the Nokia N95 used in the CAALYX system. The software uses the standard Java Application Programming Interfaces (API's) defined by the Java Collections Framework (JCF), to interact with the mobile device's resources, such as the Java Bluetooth specification (JSR 82), Java location specification (JSR 179) to access the GPS chipset of the N95, and the Java Web-Services specification (JSR 172), to interact with the Caretaker's Web services. Although defined by the Java community, the actual implementation of these API's is done by the manufacturers of the mobile devices. As a result, the phone based CAALYX software is portable across different devices. The phone based control software is responsible for data collection from the WLD, the correct inference of the user's health status from that data, and for reporting critical situations to the health server. Additionally, this software is responsible for the execution of scheduled monitoring procedures. The inference process uses medical knowledge and inference rules with threshold values that are user specific. Rules and thresholds are downloaded from the health server. In addition to reporting critical situations, this software is also responsible for detecting the position of the user using the GPS chipset of the mobile phone. The position is used to geo-reference the alerts, as well as to detect wether the user is lost or disorientated.

1) Observation Patterns and Observations: Conceptually, Observation Patterns are a procedure composed of a set of process steps, in which it is possible to define a work flow for a sensor-based application, featuring data collection, data fusion and data classification. The observation patterns are encoded using the $X M L$ language and follow an $X M L$ scheme, which has been specifically designed to be used in this prototype. This scheme uses the same concepts and types of instructions as the ones used in the Sensor Model Language [4] for the definition of process chains, but is slightly different in the actual encoding to keep overhead to a manageable size. The definition of an observation pattern may be divided in two parts: entry points and the actual process steps which define the observation work flow. The entry points can be the desired monitoring schedule for each observation, the health events that trigger a specific process chain or context restrictions. In this particular prototype, an important context restriction was the desired physical activity state to start an observation procedure, such as being at rest. The execution of an observation pattern encompasses three types of process steps: data collection, data fusion and finally

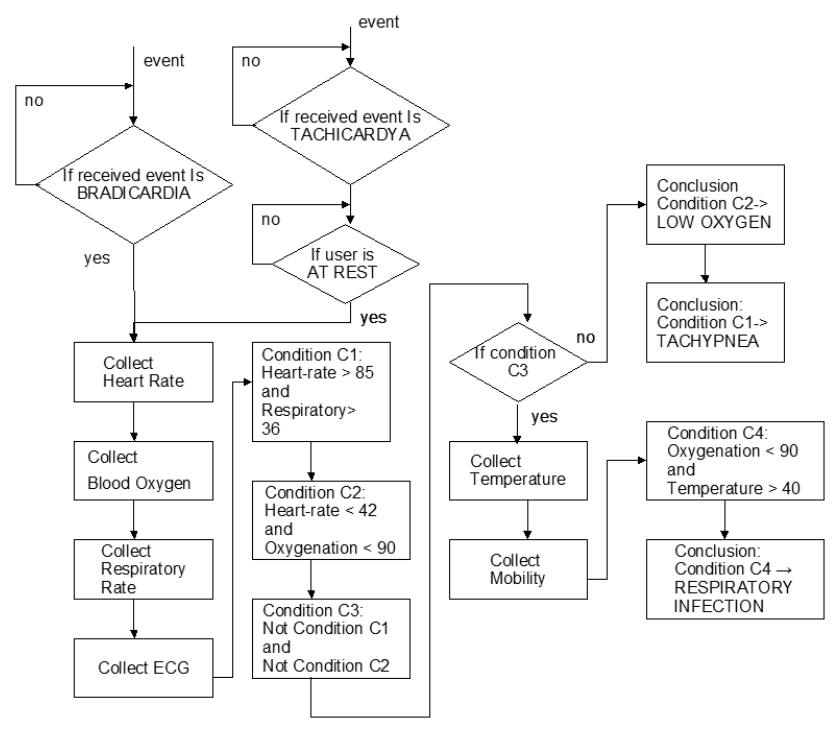

Fig. 3. An Observation pattern Workflow example

data classification. The data collection establishes which vital signs need to be collected and in what order. Observation data originating from health sensors is then fused and higher level information is extracted. The actual fusion of information is managed by a set of domain-specific axioms which build up and constitute a decision tree, relating the measured physical parameters to user-specific thresholds. Certain branches of the decision tree may trigger other process chains, in which new measurements are obtained and new rules are applied. The data classification step evaluates the conditions from the decision tree that were activated during the previous step, leading to a conclusion concerning the user's health status and corresponding priority level. In figure 3 an example of an observation pattern can be seen.

Observations are a description of a finite event. In the CAALYX system, Observations were the result of the execution of the observation pattern procedures, and they aggregated all the relevant results from such procedures, such as vital sign measurements and health conditions verified. Observations provide the following information:

- Measurements

- Conclusions

- Level of Priority

The concept of observation was adopted from the Observation Adaptive Model Pattern [5], proposed by Joseph Yoder .

2) Logical Architecture: The logical architecture of the mobile device, follows a black-board style [6]. This type of architectures is used mainly in artificial intelligence systems, and conceptually it consists of a set of independent knowledge sources, that publish facts on a central repository, where data becomes available to all knowledge sources. Once new facts are published on the repository, it notifies each of the knowledge sources about the new facts. This particular architecture was chosen to allow a hybrid event-oriented/scheduled ap- 


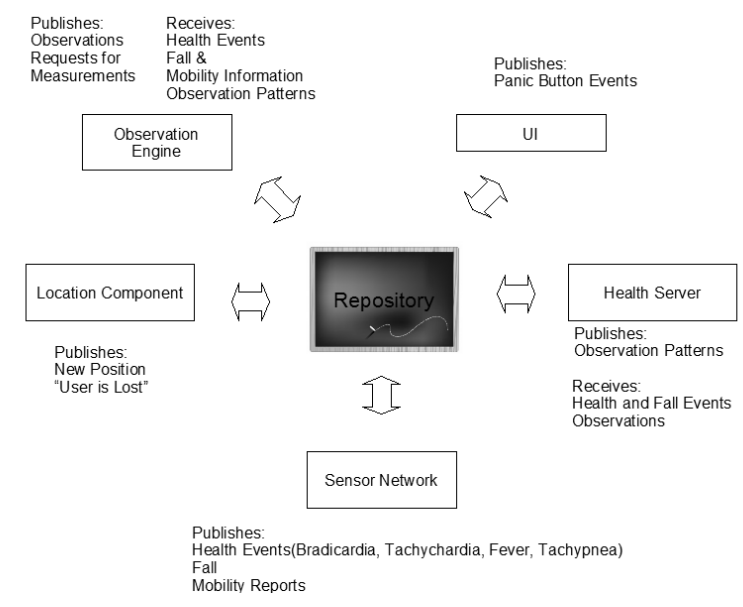

Fig. 4. Logical Architecture for the Mobile Phone Control Software

proach to save processing resources, and to allow components to be independent of each other. This independence maximizes component reusability from a development perspective, but it also improves reliability in runtime, since failures are not propagated between components. There are several knowledge sources within this software, that are responsible for handling the different resources and processing tasks:

- The Observation Engine knowledge source

- The Sensor Network knowledge source

- The Health Server Interface knowledge source

- The Location knowledge source

The Observation Engine knowledge source is the most important component in the mobile phone software, since it is responsible for all control and decision processes. The approach used relies on the execution of the work flow defined in section II-B1, which is started when health events are verified or when time-scheduled events are triggered. This way, a complete monitoring approach is provided, since the scheduled events overcome the possible incompleteness of a strictly event-driven approach. This knowledge source is able to listen to all types of relevant events of the observation patterns that are being executed and to interpret all types of steps that constitute the observations. The collection of measurements is achieved by publishing requests in the repository to obtain measurements. These requests are attended to by the sensor network knowledge source, which will publish the measurements when they are available. This component is able to follow the work flow defined by user observation patterns (section II-B1). The execution starts when health or scheduled events are posted on the repository. The scheduled events overcome the possible incompleteness of a strictly event-driven approach, since it can happen that no health events are raised and thus no observations are generated. The existence of such type of events guarantees that the minimum required health information is obtained. The observation engine is capable of executing all observation patterns. The most complex instructions are the ones that handle the mea- surement collection. Due to the fact that measurements may not be available when required, these instructions require an asynchronous mechanism. This mechanism was implemented by publishing requests in the repository, which are monitored by the sensor network. When measurements are available, the sensor network will post them back to the repository, and the measurements will be available to be used by the observation engine. To improve the effectiveness of this mechanism, a cache strategy based on time depreciation was implemented. As a result measurements can be used by different observation patterns during a certain time window.

The Sensor Network knowledge source uses the Bluetooth stack of the mobile phone to interact with the Wearable Light Device, by transmitting messages following the protocol defined. The functionalities provided by this knowledge source, are to publish the health, fall, and activity report events in the repository and configure the Wearable Light Device to report health events according to the user's specific thresholds. In addition, this knowledge source is able to receive and execute requests to collect specific measurements. After requests are received, the sensor network knowledge source interacts with the WLD to obtain the measurements, applies filtering techniques (such as averaging) over those measurements and posts the resulting data in the repository.

The Health Server knowledge source guarantees the reliable bi-directional exchange of medical information with the server. It receives Observation Patterns from the server and integrates them in the repository. Additionally it transmits all observations, fall events, health events and physical activity reports that are detected within the system.

The Location knowledge source is responsible for the interaction with the built-in GPS of the Nokia N95. It collects the GPS positions of the elderly person, and posts these positions in the repository, so that they can be attached to the alerts and observations that are transmitted to the server. This component is also responsible for all geodetic calculations necessary to detect if the user is too far away from a set of points.

3) Embedded WLD software: The software running on the WLD consists of several parts that run within a preemptive multitasking environment. The following main functional blocks can be identified:

- Main state machine and system management

- ECG recording and filtering

- Joint PPG and ECG analysis (heart rate, ptt, respiratory rate)

- Medical Event Monitor

- Communication with the pulseoximeter

- Communication with the fall sensor

- Bluetooth communication

- Power management

The Main state machine handles non medical events that affect the device behaviour including commands received by Bluetooth. Additionally the power supply and results of continuous self-test are monitored here. The recorded ECG 
is immediately filtered after recording and then written to a buffer.

Several ECG Analysis tasks read from this buffer and feed their analysis results a a set of FIFO buffers.

The Medical Event Monitor constantly compares the analysis results with configurable thresholds if the corresponding vital sign is configured for monitoring. To calculate advanced parameters like the PTT, data streams from different sources must be joined and precisely correlated. Therefore the low rate data from the pulseoximeter is tagged with high resolution time stamps to be aligned with the ECG signal. Any occurring clock skew between the different data sources is detected and corrected by comparing fill rates and fill levels in the buffers. Low level communication with the pulseoximeter, fall sensor and Bluetooth module take place in interrupt routines to keep the latency small and avoid data loss.

Bluetooth communication takes place in separate tasks. The information to be transmitted is put into a send queue and multiplexed in packets of different types before transmission to the Bluetooth communication module takes place. In order to simplify the pairing and search process of the Bluetooth connections, the WLD has the role of a slave and will accept the connection of every master that can successfully authenticate itself and has the same version number of the used transmission protocol. The fall and mobility sensor, as an independent device housed in the WLD, can utilize the Bluetooth functionality from the latter. This cable replacement feature is realized by tunnelling the data stream from the fall and mobility sensor multiplexed with all other data streams through the Bluetooth connection. A demultiplexer in the phone based software can easily extract the fall and mobility sensor's data and thus a totally independent communication with this sensor is obtained.

The Power management includes monitoring of the battery voltage and detection of a connected charger. When a charger is connected, the device will switch itself off such as to prevent confusing the external charger circuitry by drawing current other than for charging the batteries.

4) Embedded fall and mobility sensor software: The fall and mobility sensor continuously monitors the acceleration of the user. Using appropriate thresholds, both in acceleration and in time, the algorithm establishes whether the user has fallen and whether or not the user has recovered from this fall. While making its decisions, the algorithm takes into account the orientation and position of the user, the time (s)he needs to recover from a stumble and the relative time that the user spends lying, sitting or standing after a suspected fall. In addition to giving reliable information as to whether family, care givers or emergency services should be warned, the algorithm also assists health carers to assess the patient's well-being and mobility trends over a much longer timescale. This is done through the extraction of mobility data from the acceleration measurements. Using a state machine the 3D acceleration information is used to constantly track the user's current activity. This information is gathered in mobility reports, which summarise the relative time spent sitting, lying, standing and walking. These reports are sent to the mobile phone on a regular basis to aid the software on the mobile phone in choosing an appropriate scheduling of measurements. Further functionality of the fall and mobility sensor includes communication protocols using Bluetooth, a dedicated versatile high-level message protocol, a calibration facility for the onboard accelerometers and local storage of accelerometer data, mobility data and generated messages on an on-board $\mu \mathrm{SD}$ card.

\section{Clinical TRIALS}

\section{A. Clinical Trials in Limerick}

The fall and mobility sensor hardware integrated in the WLD was initially used in trials performed in Limerick (Ireland). Both fall trials and trials identifying activities of daily living (ADL) were performed. Fall trials were performed with young health subjects and ADL trials were performed with both healthy young volunteers and healthy elderly volunteers. The simulated falls study involved 10 young healthy male subjects performing simulated falls onto large crash mats. The subjects fell from a specially constructed platform under the supervision of a physical education professional. Each subject performed eight different fall types and each activity was repeated three times. The subjects ranged in age from 2435 years $(27.2 \pm 3.61$ years), body mass from 68 to $111 \mathrm{~kg}$ $(84.2 \pm 14.43 \mathrm{~kg})$, and height from 1.65 to $1.96 \mathrm{~m}(1.81 \pm 0.102$ $\mathrm{m})$. All gave written informed consent and the University of Limerick Research Ethics Committee (ULREC) approved the protocol. The simulated falls performed were: forward falls, backward falls, lateral falls left and right all performed with both legs straight and with knee flexion similar to those performed in the study by [7]. The results of these trials show excellent sensitivity of the fall algorithms for the various falls from a standing to a lying position. Only 1 fall out of a total of 240 was not identified as a fall by the fall and mobility sensor. Further trials investigated the capabilities of the fall algorithms to correctly identify various ADL as non-fall events. These trials were conducted inside the University of Limerick and in the Limerick region with ethical approval of the ULREC. In addition to the 10 young healthy subjects, the study involved 10 elderly volunteers ( $>65$ years) performing scripted ADL in their own homes. All volunteers performed 3 repetitions of 6 different ADL, thus leading to a total of 360 activities performed. Only 1 ADL was detected as a fall, which leads to an overall sensitivity of $99.7 \%$. In a further experiment, elderly volunteers used the device in an unscripted trial for a period of up to 8 hours. During this period, the elderly volunteers were free to perform their normal daily routines, whilst the fall and mobility monitor detected potential falls and extracted the user's mobility patterns. In these trials it was established that the integrated device did not obstruct the elderly user significantly in carrying out his/her daily routine.

\section{B. Clinical Trials in Ancona}

Upon the successful completion of the trials in Limerick, the CAALYX system was deployed in Ancona (Italy) and 
used by both care-home based and independently living elderly citizens. In these clinical trials, which were performed over a 3 month period, 10 elderly volunteers, 4 nurses, 2 care givers and 1 general practitioner used the system. At the same time the system was used remotely by geriatricians to monitor the trial results and provide feedback to the elder or their general practitioner if necessary.

1) SpO2: It was found that the time between inserting the finger and obtaining the first reliable reading, depends on several parameters (physical features of the user, motion of the sensor, ambient light) and was measured to be in the range of 12 seconds to 1.5 minutes. Therefore users were asked to keep their finger in the clip for about two minutes. The resulting percentage of valid measurements was $71 \%$. Additional filtering of invalid measurements on the N95 resulted in an overall percentage of measurements considered valid by the doctors of $82.4 \%$.

2) Temperature: While the general function of the temperature sensor had been proven under laboratory conditions the results in the trials showed a relatively low reliability. This was caused mainly due to the dependence of the the quality of the measurements on the immediate surroundings of the sensor. The medical validation shows that about $40 \%$ of the values can be correctly correlated to the user's body temperature.

3) Heart Rate: The percentage of heart rate measurements that were classified as valid by the WLD was $78 \%$. That means that $22 \%$ of the attempted measurements were not available (e.g. caused by low contact quality) or were disturbed by noise (e.g. generated by motion artifacts). The combination of classification in the WLD and interpretation of these results in the N95 resulted in a total percentage of heart rate measurements considered valid by doctors of $98 \%$.

4) Respiratory Rate: As reported in section II-A the respiratory rate can only be accurately measured if the subject is at rest. This was confirmed by the results from the trial. From the transmitted measurements $14 \%$ were marked as valid. From those $94.7 \%$ were rated satisfactory by the doctors in the medical validation.

5) Geo referencing: The elder's position can be requested by their caretaker through a web interface as illustrated in figure 5. During the trials in Ancona, the geo referencing system was found to be reasonably reliable. The GPS receiver in the N95 gives reliable results when the elderly user is outside. However, as expected, the GPS fix is very poor when the user is in an inside environment.

6) Qualitative feedback from users: Feedback from elderly users, geriatricians and caretakers was generally positive. Elderly users indicated to feel decidedly safer in the knowledge that the CAALYX system provides continuous monitoring of their health. Medical professionals indicated that, even though the system will not replace consults with the doctor, it does provide a useful means of obtaining a more complete assessment of the user's health.

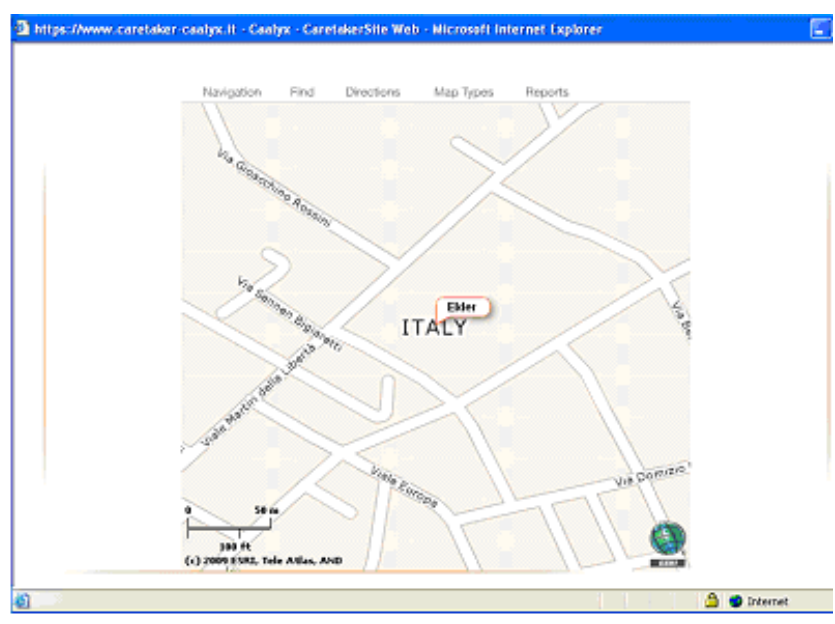

Fig. 5. Example of a geo-referenced Position

\section{CONCLUSIONS}

In this paper, the mobile sensor system of the CAALYX project was presented. This mobile sensor system consists of an ECG, SpO2, temperature, respiratory rate and fall and mobility sensor, all integrated in one device, coined the Wearable Light Device. Through a mobile phone, the device communicates with a server which allows health professionals to intervene in case of a wide variety of health threats. In addition, the software on the mobile phone uses data fusion in the scope of a decision support system to trigger additional measurements, classify health conditions or schedule future observations. Clinical trials performed with the devices were discussed and the results clearly show the possibilities and benefits of this approach.

\section{ACKNOWLEDGMENT}

This work was sponsored by the European Union under the 6th Framework Programme, Priority IST, Information Society Technologies, contract 045125 . The authors gratefully acknowledge the help and cooperation of the participants in the fall trials and the elderly volunteers in Ancona, Italy and Limerick, Ireland.

\section{REFERENCES}

[1] World demographic trends, United Nations, 2007.

[2] A. Anastasiou, P. Quarrie, and M. Boulos, "Personal location aware healthcare in europe-the challenges from prototype to product: the caalyx experience," eHealth International Journal. 2009, URL: http://www.ehealthinternational.org/vol5num1/Vol5Num1p18.pdf, vol. 12, pp. 18-29, 2009.

[3] S. R. Lord, C. Sherrington, and H. B. Menz, Falls in Older People: Risk Factors and Strategies for Prevention. Cambridge University Press, 2001.

[4] M. Botts, Ed., OpenGIS Sensor Model Language (SensorML), Implementation Specification.

[5] J. W. Yoder and R. E. Johnson, "The adaptive object-model architectural style," in WICSA 3: Proceedings of the IFIP 17th World Computer Congress - TC2 Stream / 3rd IEEE/IFIP Conference on Software Architecture. Deventer, The Netherlands, The Netherlands: Kluwer, B.V., 2002, pp. 3-27.

[6] H. P. Nii, "Blackboard systems," Stanford, CA, USA, Tech. Rep., 1986.

[7] A. Bourke, V. J. O'Brien, and G. Lyons, "Evaluation of a threshold-based tri-axial accelerometer fall detection algorithm," Gait Posture, 2006. 